\title{
A NOTE ON LIFTING BRAUER CHARACTERS
}

\author{
STEPHEN M. GAGOLA, JR.
}

ABSTRACT. A Brauer character of a finite group may be lifted to an ordinary character if it lies in a block whose defect groups are contained in a normal p-solvable subgroup.

By the Fong-Swan theorem [2, Theorem 72.1], an irreducible Brauer character of a finite $p$-solvable group $G$ may be lifted tc an ordinary (complex) character of $G$. In other words, every Brauer character $\phi$ is the restriction of some ordinary character $\chi$ to the $p$-regular elements of $G$. Professor I. M. Isaacs has shown [5] that the character $\chi$ may be chosen to satisfy certain extra conditions which when $p$ is odd, uniquely determine $\chi$. By extending a theorem which appears in that paper [5, Theorem 3.1], the hypothesis of $p$-solvability on $G$ may be weakened somewhat.

Specifically, the main result of this paper is the following

Theorem. Let $\phi$ be an irreducible Brauer character of the finite group $G$, and assume that $\phi$ lies in a block whose defect groups are contained in a normal posolvable subgroup of $G$. Then $\phi$ may be lifted to an ordinary character $X$ of $G$.

We will not be concerned with general uniqueness questions here.

For the remainder of this paper, $G$ denotes a finite group, and $F$ is a field of characteristic $p$ which is a splitting field for all subgroups of $G$. If $V$ is an $F[G]$-module, let $J(V)$ be the intersection of all maximal submodules of $V$. Finally, if $U$ and $V$ are $F[G]$-modules affording the Brauer characters $\phi$ and $\mu$, respectively, and if $V$ is irreducible, then the multiplicity of $\mu$ in $\phi$ is the multiplicity of $V$ as a composition factor of $U$.

Lemm a 1. Let $N \triangleleft G$ and let $W$ be an irreducible $F[N] \cdot$ module which affords the Brauer character $\mu$. Assume that $\mu$ can be lifted to an ordinary character $\Psi$ in such a way that the inertia groups ${ }_{G}(\mu)$ and $\mathscr{I}_{G}(\Psi)$ coincide. Denote by $\mu^{G}$ the Brauer character which the induced module $W^{G}$ affords. Let $\mathcal{S}$ denote the set of all irreducible Brauer characters $\phi$ of $G$ which are constituents of $\mu^{G}$, but which are not afforded by any composition factor of $J\left(W^{G}\right)$. Finally, let $\mathcal{T}$ denote the set of ordinary irreducible characters $x$ of $G$ which are constituents of $\Psi^{G}$ and which have the property that the

Received by the editors December 24, 1974.

AMS (MOS) subject classifications (1970). Primary 20C20.

Key words and phrases. Lifting Brauer characters, vertices, defect groups. 
decomposition number $d_{\boldsymbol{\chi} \phi}$ does not wanish for some $\phi \in \mathcal{S}$. Then, restriction to p-regular elements is a 1-1 correspondence between the elements of $\mathcal{T}$ and the elements of $\mathfrak{S}$.

Proof. Write $\mu^{G}=\Sigma_{\phi \epsilon \mathcal{S}} f_{\phi} \phi+\Phi$, where no constituent of $\Phi$ lies in $\mathcal{S}$, We first compute the restriction of $\phi$ to $N$, where $\phi \in \mathcal{S}$. Let $W$ and $V$ be irreducible $F[N]$ - and $F[G]$-modules affording $\mu$ and $\phi$, respectively. By Clifford's theorem, $V_{N}$ is completely reducible and, since $F$ is a splitting field for $N$, the multiplicity of $W$ as a composition factor of $V_{N}$ is the $F$-dimension of hom $\left.F_{[N}\right]^{\left(W, V_{N}\right)}$. By the Nakayama relations $[4, \mathrm{p} .556]$, this di-

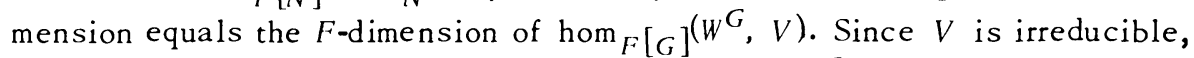

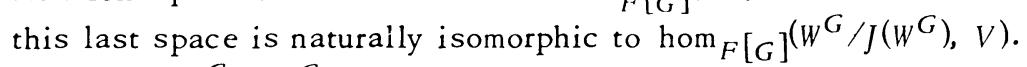

Since $W^{G} / J\left(W^{G}\right)$ is completely reducible, and $F$ is a splitting field for $G$, this last space has $F$-dimension equal to the multiplicity of $V$ in $W^{G} / J\left(W^{G}\right)$. However, $\phi \in \mathcal{S}$, which means that $V$ is not a composition factor of $J\left(W^{G}\right)$, so that the multiplicity of $V$ in $W / J\left(W^{G}\right)$ is $f_{\phi}$. Therefore, $\mu$ appears in $\phi_{N}$ with multiplicity $f_{\phi}$. We may write

$$
\phi_{N}=f_{\phi}\left(\mu_{1}+\cdots+\mu_{t}\right) \text {, }
$$

where $\mu=\mu_{1}$ and $\mu_{1}, \ldots, \mu_{t}$ are the distinct $G$-conjugates of $\mu_{\bullet}$

Similarly write $\Psi^{G}=\Sigma e_{\chi} \chi+X$, where each $\chi$ lies in $\mathcal{T}$, and no constituent of $X$ lies in $\mathcal{T}$. Since ${ }_{G}(\mu)={ }_{G}(\Psi)$, the number of distinct $G \cdot$ conjugates of $\Psi$ is $t$, and by Frobenius reciprocity,

$$
\chi_{N}=e_{\chi}\left(\Psi_{1}+\cdots+\Psi_{t}\right)
$$

where $\Psi=\Psi_{1}, \Psi_{2}, \ldots, \Psi_{t}$ are the distinct $G$-conjugates of $\Psi$.

Let $R$ and $S$ denote the set of $p$-regular elements of $G$ and $N$, respectively. We now use the equations

$$
\left(\left.\Psi\right|_{S}\right)^{G}=\left(\Psi^{G}\right)_{R} \quad \text { and } \quad\left(\left.\chi\right|_{R}\right)_{N}=\left.\left(\chi_{N}\right)\right|_{S} .
$$

(The first equation follows from the fact that the values of $\mu^{G}$ may be computed by the usual formulas for an induced class function, a fact proved in $[1, \S 25]$.) The first equation may be rewritten as

$$
\sum_{\phi \in \mathcal{S}} f_{\phi} \phi+\Phi=\sum_{\chi \in \mathcal{J}} e_{\chi} \chi_{R}+X_{R}
$$

This implies that for $x \in \mathcal{T}$,

$$
\chi_{R}=\sum_{\phi \in \boldsymbol{\delta}} d_{\boldsymbol{x} \phi} \phi+\eta_{\boldsymbol{\chi}}
$$

where $\eta_{\boldsymbol{\chi}}$ has constituents appearing in $\Phi$. Now, no $\phi$ in $\mathcal{S}$ appears as a constituent of $X_{R}$, so that

$$
f_{\phi}=\sum_{\boldsymbol{x} \in \mathcal{T}} e_{\boldsymbol{\chi}} d_{\mathbf{x} \phi^{\cdot}}
$$


For $\chi \in \mathcal{T}$, the equation $\left(\left.\chi\right|_{R}\right)_{N}=\left.\left(\chi_{N}\right)\right|_{S}$ implies

$$
\sum_{\phi \in \mathcal{S}} d_{\boldsymbol{x} \phi} \phi_{N}+\left(\eta_{\chi}\right)_{N}=e_{\chi}\left(\mu_{1}+\cdots+\mu_{t}\right),
$$

and since $\phi_{N}=f_{\phi}\left(\mu_{1}+\cdots+\mu_{t}\right)$, we get

$$
e_{\chi} \geq \sum_{\phi \in \mathcal{S}} d_{x \phi} f_{\phi}
$$

Now, combining $(*)$ and $(* *)$ :

$$
\begin{aligned}
& f_{\phi}=\sum_{\boldsymbol{\chi} \in \mathcal{T}} e_{\boldsymbol{\chi}} d_{\boldsymbol{\chi} \phi} \geq \sum_{\boldsymbol{\chi} \in \mathcal{S}} \sum_{\phi^{\prime} \in \mathcal{S}} d_{\boldsymbol{\chi}} \phi_{\phi^{\prime}} f_{\phi^{\prime}} d_{\boldsymbol{\chi} \phi} \\
& =\sum_{\phi^{\prime} \in \mathcal{S}}\left(\sum_{\boldsymbol{\chi} \in \mathcal{T}} d_{\boldsymbol{\chi}} \phi^{\prime} d_{\boldsymbol{\chi} \phi}\right) f_{\phi^{\prime}} \\
& >\left(\sum_{\chi \in \mathcal{T}} d_{\chi \phi}^{2}\right) f_{\phi} \geq f_{\phi} \text {. }
\end{aligned}
$$

The last inequality is valid since $(*)$ implies that, for every $\phi \in \mathcal{S}$, there exists $\chi \in \mathcal{T}$ with $d_{\boldsymbol{x} \phi} \neq 0$. We now have that equality holds throughout, in the above chain of inequalities, and, in particular, $\Sigma_{\chi \in \mathcal{J}} d_{\chi \phi}^{2}=1$ holds for every $\phi \in \mathcal{S}_{\text {。 }}$ Therefore, for every $\phi \in \mathcal{S}$, there exists a unique $\chi \in \mathcal{T}$ with $d_{\boldsymbol{x} \phi}=1$, and $d_{\chi^{\prime} \phi}=0$ for $\chi^{\prime} \neq \chi$ in $\mathcal{T}$. But then $(*)$ implies that $f_{\phi}$ $=e_{\chi}$, and since $\mu(1)=\Psi(1), \chi$ must be a lift of $\phi$. We have now proved that every $\phi \in \mathcal{S}$ has a unique lift $\chi$ in $\mathcal{T}$. Finally, if $\chi \in \mathcal{T}$, then, by definition, there exists $\phi \in \mathcal{S}$ with $d_{\chi \phi} \neq 0$. But, by the above, $\chi$ is a lift of $\phi$. Thus, the map $\chi \mapsto \chi_{R}$ is a 1-1 correspondence between $\mathcal{T}$ and $\mathcal{S}$.

Definition. Let $V$ be an $F[G]$-module and $N$ a subgroup of $G . V$ is $N$. reducible if every exact sequence of $F[G]$-modules $U \mapsto V \rightarrow Y$, which splits when considered as a sequence of $F[N]$-modules, necessarily splits as a sequence of $F[G]$-modules. Thus, every module is $G$-reducible, and a module is 1-reducible iff it is completely reducible. (By using the other two positions of the exact sequence, one can define the usual notions of $\mathrm{N}$-injectivity and $N$-projectivity.)

Lemma 2. Let $V$ be an $F[G]$-module and $N$ a subgroup of $G$. Let $T$ be a set of coset representatives for the right cosets of $N$ in $G$. Assume that there exists a $\in C_{F[G]}(N)$ such that $\Sigma_{x \in T} x^{-1} a x$ acts like the identity on $V$. Then $V$ is N-reducible.

Proof. This is essentially the proof of $(d) \rightarrow(a)$ of Theorem 1 of [3].

Lemma 3. Let $N \triangleleft G$ and let $\mu$ be an irreducible Brauer character of $N$. Assume that $\mu$ can be lifted to an ordinary character $\Psi$ with the property that $g_{G}(\mu)=9_{G}(\Psi)$. Finally, let $B$ be a p-block of $G$ whose defect groups are contained in $N$. Then the restriction to p-regular elements defines a 
1-1 correspondence between the set of irreducible constituents of $\Psi^{G}$ which lic in $B$ and the set of irreducible Brauer constituents of $\mu^{G}$ which lie in $B$.

Proof. Define $\mathcal{S}$ and $\mathcal{S}$ as in the statement of Lemma 1, and again let $R$ denote the set of $p$-regular elements of $G$. Then $\chi \mapsto \chi_{R}$ is a 1-1 correspondence between the elements of $\mathcal{d}$ and $\zeta$. Clearly $\chi \in B$ iff $\chi_{R} \in B$. It suffices to show that all the irreducible Brauer constituents of $\mu^{G}$ which belong to $B$ necessarily lie in $\mathcal{S}$.

Let $W$ afford $\mu$ and let $\rho$ denote the centrally primitive idempotent of $F[G]$ which corresponds to the block $B$. Then $W^{G}=\left(W^{G}\right)_{e}+\left(W^{G}\right)(1-e)$. The composition factors of $\left(W^{G}\right) e$ afford Brauer characters in $B$, and no composition factor of $\left(W^{G}\right)(1-e)$ belongs to $B$. We must show that $J\left(W^{G}\right)$ $\subseteq\left(W^{G}\right)(1-e)$, and this is equivalent to the statement that $\left(W^{G}\right) e$ is complete= ly reducible.

Since $B$ has a defect group contained in $N$, it follows that the block idempotent $e$ has a representation of the form $e=\Sigma_{x \in T} x^{-1} \alpha x$, where $T$ is a set of coset representatives for $N$ in $G$, and $a \in C_{F[G]}(N)$. (This is essentially Lemma 54.8 of $\left[2, p_{0} 346\right]$ with $F$ in place of $R_{0}$ )

Thus, $\left(W^{G}\right) e$ is $N$-reducible by Lemma 2 . Therefore, $\left(W^{G}\right) e$ is completely reducible as an $F[G]$-module iff $\left.\left(W^{G}\right) e\right|_{N}$ is completely reducible as an $F[N]$-module. However $\left.\left(W^{G}\right) e\right|_{N}$ is a summand of $\left(W^{G}\right)_{N}=\Sigma_{x \in T} \cdot W \otimes x$ where each $W \otimes x$ is simple. Hence, $\left(W^{G}\right) e$ is completely reducible, and we are done.

In order to prove the main theorem of this paper, we need the strengthened version of the Fong-Swan theorem appearing in [5].

Lemma 4. Let $N$ be a p-solvable group and $\mu$ an irreducible Brauer character of $N$. Then there exists an ordinary irreducible character $\Psi$ of $N$ which lifts $\mu$ and satisfies $\mathfrak{I}_{A}(\mu)=9_{A}(\Psi)$, where $A$ is the automorphism group of $N$.

Proof. This is contained in Theorem 5.4 of [5].

We now present a proof of the theorem quoted at the beginning of the paper.

Let $\phi$ be an irreducible Brauer character of $G$ and assume that $\phi$ lies in a block whose defect groups are contained in the normal $p$-solvable subgroup $N$. Let $\mu$ be a constituent of $\phi_{N}$ and lift $\mu$ to an ordinary character $\Psi$ satisfying the conclusion of Lemma 4. Since $G$ induces on $N$ a group of automorphisms, clearly $\mathfrak{I}_{G}(\mu)=\mathfrak{I}_{G}(\Psi)$. Lemma 3 now implies that $\phi$ has a lift (which is a constituent of $\Psi^{G}$ ).

We remark that by replacing $N$ by the largest normal $p$-solvable subgroup of $G$ (so as to assume that $N$ is characteristic in $G$ ), it is easy to show that $\phi$ has a lift $\chi$ which satisfies $g_{\mathrm{Aut}(G)}(\phi)=g_{\mathrm{Aut}(G)}(\chi)$. 
Closing remarks. I would like to thank the referee for his careful reading of this paper and for pointing out that, in the situation of Lemma 1, the set $\mathcal{S}$ consists precisely of those irreducible Brauer constituents of $\mu^{G}$ which have vertices contained in $N$. (Of course, the vertex of a Brauer character means the vertex of an irreducible $F[G]$-module which affords it.) This observation follows from the equivalence of (i) and (iii) in the following

Proposition. Let $N \triangleleft G, W$ an irreducible $F[N]$-module and $V$ an irreducible $F[G]$-module. Assume that $V$ is a composition factor of $W^{G}$. Then the following conditions on $V$ are equivalent.

(i) A vertex for $V$ is contained in $N$.

(ii) $W^{G}=U$ iS where no composition factor of $U$ is isomorphic to $V$, and $S$ is a direct sum of simple modules all being isomorphic to $V$.

(iii) $V$ is not a composition factor of $J\left(W^{G}\right)$.

Proof. (i) $\rightarrow$ (ii). Write $W^{G}=U \mp S$ with $S$ isomorphic to a direct sum of copies of $V$ and with $\operatorname{dim}_{F} U$ minimal. Suppose $X$ and $Y$ are submodules of $U$ with $Y \leq X$ and $X / Y \cong V$. Then $Y \mapsto X \rightarrow V$ is an exact sequence of $F[G]$-modules. Since $X$ is a submodule of $W^{G}$, and $\left.W^{G}\right|_{N}$ is completely reducible, the sequence splits when regarded as a sequence of $F[N]$-modules. However, a vertex of $V$ is contained in $N$, so $V$ is $N$-projective and the sequence splits as a sequence of $F[G]$-modules. Thus $V$ is isomorphic to a submodule $V_{1}$ of $X$ and, hence, of $U$. By considering the sequence $V_{1} \hookrightarrow$ $U \rightarrow U / V_{1}$ and using the fact that $V_{1}$ is $N$-injective, we have $U=U_{0}+V_{1}$. But then $W^{G}=U_{0}+\left(V_{1}+S\right)$, contradicting the minimality of $\operatorname{dim}_{F} U$, and thereby proving (ii).

(ii) $\rightarrow$ (iii). The hypothesis of (ii) implies $J\left(W^{G}\right) \subseteq U$ and (iii) is immediate.

(iii) $\rightarrow$ (i). Since $V$ is a composition factor of $W^{G}$, it follows from the Nakayama relations and the semisimplicity of $V_{N}$ that $W$ is a summand (and, hence, a homomorphic image) of $V_{N}$. By the Nakayama relations again, $V$ is isomorphic to a submodule, say $V_{1}$, of $W^{G}$. Since $V_{1} \unrhd J\left(W^{G}\right)$, we have $W^{G}=V_{1}+M$ for some maximal submodule $M$ of $W^{G}$. This equation implies that $V_{1}$ is $N$-projective, and so $N$ contains a vertex of $V_{1}$, proving (i).

As the referee has kindly pointed out, this Proposition, together with Lemmas 1 and 4 imply the following strengthened version of the main theorem of this paper:

Theorem. Let $\phi$ be an irreducible Brauer character of $G$ and assume that a vertex for a module affording $\phi$ is contained in a normal p-solvable subgroup of $G$. Then $\phi$ may be lifted to an ordinary character $\chi$ of $G$.

\section{REFERENCES}

1. R. Brauer and C. Nesbitt, On the modular characters of groups, Ann. of Math. (2) 42 (1941), 556-590. MR 2, 309. 
2. L. Dornhoff, Group repre'sentation the'ory. Part B, Marcel Dekker, New York, 1972.

3. D. G. Higman, Modules with a group of operators, Duke Math. J. 21 (1954), 369-376. MR 16, 794.

4. B. Huppert, Endliche Gruppen. I, Die Grundlehren der math. Wissenschaften, Band 134, Springer-Verlag, Berlin and New York, 1967. MR 37 \#302.

5. I. M. Isaacs, Lifting Brauer characters of pesolvable groups, Pacific J. Math. 53 (1974), 171-188.

DEPARTMENT OF MATHEMATICS. MICHIGAN STATE UNIVERSITY, EAST LANSING, MIC.HIGAN 48824 